Revista de la Facultad de Medicina de la Universidad de lberoamérica
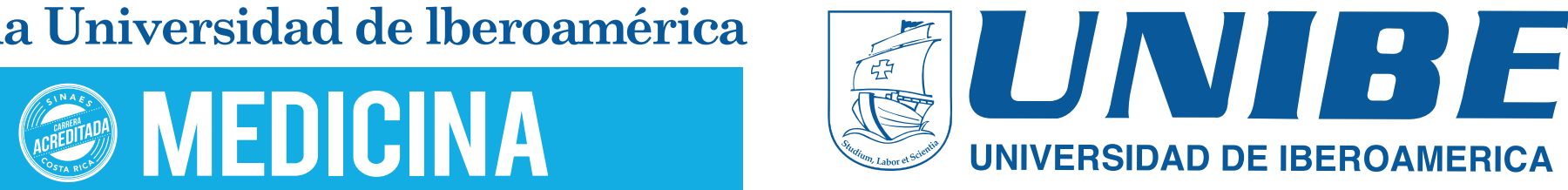

\title{
Articulo
}

\section{Feminización de la Carrera de Medicina: Facultad de Medicina de la Universidad de Iberoamerica 1995-2015}

Dra. Maria del Rosario Calvo Fonseca

Dr. Juan Ignacio Padilla Cuadra

\section{Resumen}

Introducción: Las desigualdades en el acceso a la educación superior se deben a actores geográficos, económicos-sociales, religiosos, étnicos y género. La carrera de medicina ha mostrado recientemente una tendencia al predominio del sexo femenino entre sus estudiantes y graduados, fenómeno denominado feminización de esta carrera.

\section{Metodología}

Se realizó estudio de la base de datos de la población estudiantil y graduados de la carrera de medicina de la Universidad de Iberoamérica en el periodo de tiempo comprendido entre 1995-2015.

\section{Resultados}

Se determinó que, de un total de 4974 matriculados en el periodo de estudio, 3109 $(62.5 \%)$ son de sexo femenino y 1865 (37.5\%) de sexo masculino. Durante el periodo 1995val 2015, la relación entre estudiantes de sexo femenino y masculino cambió en 1995 de 1.13 a 2.13 para el 2015 .

\section{Conclusiones}

Se demuestra que hay una tendencia a la feminización en la población estudiada. Este fenómeno de feminización es evidente y puede tener implicaciones en el futuro en relación con la disponibilidad de especialistas en áreas en las que las mujeres no incurren con frecuencia.

\section{Palabras Clave}

Feminización, Carrera de medicina

\section{Abstract}

Introduction: The differences on access to high education depend on geographic, economic, social religious, ethnic and gender factors. Recently, medical career has shown a trend to female predominance, this phenomenon is called feminization of medicine.

\section{Methods}

We review the database on students and graduates of medical school of the Universidad de Iberoamérica during 1995-2015. 
Results: There was found that from a total of 4974 medical students during the study period, 3109 (62.5\%) were female and 1865 (37.5\%) were males. The ratio between females and males changed from 1995 with 1.13 to 2.13 in 2015 .

\section{Conclusions}

There is trend during the period studied toward feminization of the medical career. Feminization is clear at it may have future implications on specialist availability on areas where women are not interested.

\section{Keywords}

Feminization, Medical career

\section{Introducción}

El acceso a la educación superior se encuentra condicionado a diversos factores geográficos, económicos-sociales, religiosos, étnicos y género (1). De los anteriores factores, el género ha marcado importantes diferencias en cuanto a la proporción entre hombres y mujeres que ingresan a las diversas carreras universitarias.

Históricamente, el sexo masculino ha sido predominante en diferentes carreras universitarias incluyendo medicina. No obstante, en años recientes, esta predominancia ha tendido a invertirse, siendo ahora mayor la cantidad de mujeres que se matriculan y se gradúan de esta carrera.

El fenómeno, denominado feminización de la medicina, no es simplemente un asunto de género, sino que tiene impacto en aspectos laborales de esta profesión e incluso en la disponibilidad de ciertas especialidades de dominio masculino.

Debido a las implicaciones de este fenómeno se decidió realizar estudio sobre éste en una población de estudiantes y graduados de esta carrera a lo largo de casi 20 años en nuestra universidad.

\section{Metodología}

Se propone como objetivo principal la caracterización de la población estudiantil matriculada en la carrera de medicina y los graduados de esta facultad desde su fundación en 1995.

Para obtener los datos necesarios se acceso de las siguientes estadísticas de diferentes fuentes distribución de estudiantes matriculados distribuidos por genero según el Departamento de Registro de la universidad en los grados de bachillerato y licenciatura entre 1997 - 2014.

La información obtenida por tabulada en hoja de cálculo EXCEL 2013 (Microsoft) para el análisis y graficación de los datos Resultados Se determinó que desde 1995, la facultad de medicina ha acumulado un total de 4974 estudiantes matriculados.

Al analizar la evolución, la población matriculada ha sido siempre la mayoría de sexo femenino (cuadro 1 y cuadro 2). Se muestra un aumento en esta predominancia en tanto la relación entre estudiantes de sexo femenino en relación con el masculino para 1995 fue de 1.13 y de 2.13 para el 2015 .

Para determinar si efectivamente hay un predominio del género femenino en la carrera, en primer lugar, se contrastó si las diferencias que se observan entre ambos géneros son atribuibles al azar.

En este caso utilizando el programa SPSS se obtuvo una significación cercana al $0 \%$ con lo que para el nivel de significación habitual del $5 \%$, se rechaza la hipótesis de independencia de las prioridades de los estudiantes y el género (las preferencias no se distribuyen del mismo modo entre hombres y mujeres). Por tanto, concluyendo que si hay un predominio estadísticamente significativo del sexo femenino. 
Cuadro 1. Distribución según género de matriculados en bachillerato en medicina $\mathrm{y}$ cirugía (valores absolutos).

UNIBE. Cohortes 1995 al 2015

\begin{tabular}{|lcl|}
\hline Año & Hombres & Mujeres \\
\hline 1995 & 119 & 135 \\
1996 & 91 & 156 \\
1997 & 78 & 117 \\
1998 & 86 & 118 \\
1999 & 55 & 75 \\
2000 & 33 & 65 \\
2001 & 72 & 105 \\
2002 & 60 & 104 \\
2003 & 81 & 98 \\
\hline 2004 & 63 & 108 \\
2005 & 49 & 73 \\
2006 & 58 & 88 \\
2007 & 54 & 106 \\
\hline 2008 & 95 & 184 \\
\hline 2009 & 118 & 224 \\
\hline 2010 & 146 & 268 \\
2011 & 168 & 250 \\
\hline 2012 & 129 & 243 \\
\hline 2013 & 144 & 251 \\
\hline 2014 & 115 & 232 \\
2015 & 51 & 109 \\
\hline
\end{tabular}

Fuente: Departamento de Registro, Universidad de Iberoamérica, UNIBE
Cuadro 2. Distribución según género de matriculados en Licenciatura en medicina y cirugía. (Valores Relativos).

Facultad de Medicina, UNIBE, UNIBE, Cohortes 1995 al 2015

\begin{tabular}{|ccc|}
\hline Año & Hombres & Mujeres \\
\hline 1995 & 46,9 & 53,1 \\
1996 & 36,8 & 63,2 \\
1997 & 40,0 & 60,0 \\
1998 & $41 ., 3$ & 58,7 \\
1999 & 42,3 & 57,7 \\
\hline 2000 & 33,7 & 66,3 \\
2001 & 40,7 & 59,3 \\
2002 & 36,6 & 63,4 \\
\hline 2003 & 45,3 & 54,7 \\
\hline 2004 & 36,8 & 63,2 \\
2005 & 40,2 & 59,8 \\
2006 & 39,7 & 60,3 \\
2007 & 33,8 & 66,3 \\
\hline 2008 & 34,1 & 65,9 \\
\hline 2009 & 34,5 & 65,5 \\
\hline 2010 & 35,3 & 64,7 \\
2011 & 40,2 & 59,8 \\
\hline 2012 & 34,7 & 65,3 \\
\hline 2013 & 36,5 & 63,5 \\
\hline 2014 & 33,1 & 66,9 \\
2015 & 31,9 & 68,1 \\
\hline
\end{tabular}

Fuente: Departamento de Registro, UNIBE 


\section{Discusión}

Hasta recientemente, la incorporación de las mujeres en campos como la academia, la ciencia y la tecnología era muy limitada (2).

Muchos factores fueron determinantes para tal situación incluyendo prejuicios y discriminación. Fue hasta la transición de entre el siglo XIX y $\mathrm{XX}$ que hubo cambios de índole legal que propiciaron la educación de las mujeres. La instauración de gobiernos con políticas de igualdad y de promoción de los estudios superiores, difusión de la educación femenina, el carácter gratuito de la educación y la influencia de modelos culturales que incorporan cada vez la educación como una meta han beneficiado la mayor incursión de la mujer en la educación universitaria (3).

Por ejemplo, en 1872, se registra el ingreso de la primera mujer en la Facultad de Medicina de Barcelona (4). En el caso de Costa Rica, la primera mujer inscrita como médico fue la doctora Isabel Calderón Rojas quien nació en 1902, y corresponde al décimo séptimo médico registrado en nuestro país. Este proceso de incorporación de la mujer a la medicina se extendió en los años siguientes a Suiza, Inglaterra, Francia y Alemania (2). En México, Matilde Montoya fue la primera graduada de la escuela de medicina en 1887 (5).

Esta aproximación de la mujer a la carrera de medicina fue aumentada hasta en las últimas décadas ha llegado a invertir la predominancia a un número de estudiantes de sexo femenino que supera a los de sexo masculino (6).

De acuerdo con Bonilla y colaboradores, al revisar informes sobre la participación de la mujer en la educación superior de 18 países de Latinoamérica, con excepción de Chile, Bolivia y Perú, para el periodo de 1995-2014 el porcentaje de mujeres matriculadas en las universidades supera a los hombres (7).
En comparación con la selección de carreras, estos investigadores muestran como las mujeres predominan en el área de educación, ciencias sociales y ciencias de la salud. Un perfil similar se repite para las mujeres en cunado a los egresos de la carrera. En el caso de los hombres, la selección de carrera es principalmente hacia ingeniería, ciencia y tecnología y las ciencias agrícolas o agropecuarias.

Para explicar este tipo de escogencia de carrera en las mujeres, Sierra Escalonada indica puede responder a una prolongación de la condición natural de la mujer por cuidar y educar a otros (8). Además, se atribuye el aumento del ingreso a mujeres a ciertas carreras implica la elección de carreras con mayor compatibilidad con sus roles domésticos y públicos. Esto contrasta con los de ciencias fisicomatemáticas e ingenierías donde siguen superando en número los hombres a las mujeres lo cual se extiende los estudios de posgrado. Tal como indican Garay y Valle-Díaz-Muñoz, en México, la participación de las mujeres en carreras ingeniería y tecnología se encuentra por debajo de la mitad, mientras que, en las áreas de ciencias de la salud, ciencias sociales, administrativas, educación y humanidades superan el $50 \%$ de la matrícula (9). Para Miroiu, este fenómeno puede tener efectos negativos ya que puede producirse un sobre oferta de egresados en ciencias sociales pero una carencia de profesionales en áreas científicas (10).

Entre las ventajas de la incorporación de la mujer a la carrera de medicina se destacan algunas particularidades de su relación con el paciente. $\mathrm{Al}$ respecto, en un informe elaborado por el General Medical Council del Reino Unido en el 2013 sobre el estado de la educación y práctica médica, se documenta se reciben más quejas sobre el ejercicio que los médicos de sexo masculino que de los de sexo femenino (11). Esto podría ser producto de una mejor comunicación entre las profesionales con sus pacientes. 
Sin embargo, se ha mencionado que la tendencia de la mujer hacia ciertas especialidades y su predominancia en la carrera podría acarrear una reducción en la disponibilidad de ciertos especialistas. En relación con la escogencia de especialidad, es importante reconocer que algunas especialidades imponen por su naturaleza ciertas limitaciones para las mujeres. La posibilidad de embarazo reduce el ingreso a una especialidad quirúrgica, lo mismo que la exposición a agentes nocivos como anestésicos, infecciones o radiaciones reducen la incursión en especialidades quirúrgicas, anestesiología y radiología. Tanto así que, en Estados Unidos, solo el $1 \%$ de los estudiantes de especialidad quirúrgica son mujeres (1).

Según Alers et al, en una cohorte de 612 estudiantes de medicina, las mujeres prefieren escoger especialidades jornadas de medio tiempo que le permitan disponer de tiempo en el cuidado de su familia. Además, este mismo autor en otro estudio similar demuestra que la cirugía sigue siendo una especialidad de predomino masculino mientras que la inecología, pediatría y medicina familiar de predomino femenino (14). Por su parte, Riepen y colaboradores, recalcan similar predomino de las mujeres en la especialidad de ginecología (15).

Buddenberg et al demuestran mediante sondeo que, en efecto, las mujeres tienden a escoger jornadas parciales (16). Sin embargo, a pesar de lo anterior, no puede afirmarse que el proceso de feminización sea el responsable de falta de disponibilidad de personal especializado en ciertas áreas.

Finalmente, debe también aclararse, que, a pesar del aumento de graduados del sexo femenino en esta carrera, no siempre esto no siempre significa una equidad en ingresos ni posición de liderazgo ni una carrera académica asegurada. A pesar de esta feminización de la carrera, el aumento en el número de mujeres graduadas no se ha equiparado con mejores ingresos ya que estas diferencias persisten en relación con los hombres (17). De igual manera, no ha aumentado en proporción las mujeres que logran puestos de liderazgo (18) (19) (21). Un porcentaje superior al $90 \%$ de los puestos de rectoría y direcciones de divisiones académicas están ocupados por profesionales de sexo masculino (9).

De igual manera, de acuerdo con estudios de la Organización Internacional del Trabajo (OIT) hay desigualdades importantes en relación con las oportunidades laborales de las mujeres en donde ellas requieren credenciales significativamente suprior para acceder a las mismas oportunidades de empleo que los hombres (20).

En conclusión, el presente estudio demuestra, de manera similar a otros países, que en nuestra población de estudiantes y graduados de la carrera de medicina se nota un proceso de feminización en el periodo de tiempo estudiado. El impacto de este proceso feminización sobre la disponibilidad de especialistas en áreas en las que las mujeres no incurren con frecuencia solo puede determinarse mediante un estudio posterior.

De igual forma, debe investigarse en nuestro medio, si a pesar de una mayor participación de la mujer en la carrera de medicina se mantiene una incursión menor en puestos de liderazgo y en la academia. 


\section{Referencias}

1. Ovando C, Cristina K. Género y educación superior. Mujeres en la docencia y administración en las instituciones de educación superior.

En publicación: Escenarios mundiales de la educación superior. Análisis global y estudios de casos. López Segrera, Francisco. CLACSO, Consejo Latinoamericano de Ciencias Sociales. 2007 143-154.

2. Sánchez-Guzmán MA, Corona-Vázquez T. Inserción de las mujeres en la ciencia. Gac Méd Méx 2009; 145 (1):71-76.

3. Palermo I. La educación universitaria de la mujer entre las reivindicaciones y las realizaciones Revista Alternativas: Serie Historia y prácticas pedagógicas, Facultad de Ciencias Humanas Universidad de San Luis. 2000; 3(3): 1-6.

4. Benso C. Exclusión, discriminación y resistencias: El acceso de la mujer al sistema educativo (1833-1930).

Disponible en http://webs.uvigo.es/pmayobre/ master/textos/benso/mujer_y_educacion.doc

5. Cristal S, Esquivel G, Romo E. Bosquejo histórico de la participación de las mujeres de México en ciencias e ingeniería. Departamento de Ingeniería Eléctrica y Electrónica, Tecnológico de Monterrey Campus Ciudad de México, México D.F.

Disponible en http://www.sosask.ieee.ca/reg/9/ documentos/files/ wie-concurso/Garibo-

Romo.pdf

6. Alcántara A. Tendencias mundiales de la educación superior: El papel de los organismos multilaterales,

http://www.ses.unam.mx/integrantes/alcantara /publicaciones/Tendencias.pdf,

Artículo del CEIICH, UNAM, 2006.
7. Bonilla VE, López A, Cintrón M, Ramírez S, Román R. Feminización de la matrícula de educación superior en Puerto Rico. Cuaderno de Investigación en la Educación 2005; 20: 7-26.

8. Sierra R. Feminización de la matrícula de educación superior y mercado de trabajo en Venezuela: 1970-1999. Del Instituto Internacional para la Educación Superior en América Latina y el Caribe. 2003 disponible en http://www.iesalc.unesco.org.ve/

9. De Garay A. Valle-Díaz-Muñoz G, (2011), "Una mirada a la presencia de las mujeres en la educación superior en México", en Revista Iberoamericana de Educación Superior (RIES), México, UNAM-IISUE/Universia, vol. III, núm. 6 , link [consulta: fecha de última consulta].1-41

10. Miroiu, M. (2003), Guidelines for promoting gender equity in higher education in Central and Eastern Europe, Bucarest, CEPES/UNESCO.

11. General Medical Council. The state of medical education and practice in the UK. 2013, pp 1-109.

12. Cortés-Flores, A. et al. (2005), "Medicina académica y género. La mujer en especialidades quirúrgicas", en Gaceta Médica de México, México, vol. 141, núm. 4, México.

13. Alers M, Verdonk P, Bor H, Hamberg K, Lagro-Janssen A. Gendered career considerations consolidate from the start of medical education. Int J Med Educ. 2014 ;5: 178-84.

14. Alers $\mathrm{M}$, van Leerdam L, Dielissen $\mathrm{P}$, Lagro-Janssen A. Gendered specialities during medical education: a literature review. Perspect Med Educ. 2014 ;3(3):163-78. 
15. Riepen T, Möbus V, Kullmer U, Tinneberg HR, Münstedt K. Male and Female Physicians in Hospital Gynaecology Departments Analysis of the Impact of "Feminisation" from the Viewpoint of Medical Directors. Geburtshilfe Frauenheilkd. 2013;73(1):53-58.

16. Buddeberg-Fischer B1, Stamm M, Buddeberg C, Bauer G, Häemmig O, Knecht M, Klaghofer R.The impact of gender and parenthood on physicians' careers--professional and personal situation seven years after graduation. BMC Health Serv Res. 2010;10:40.

17. Puljak L, Kojundzic SL, Sapunar D. Gender and academic medicine: a good pipeline of women graduates is not advancing. Teach Learn Med. 2008;20(3):273-8.

18. Flores-Domínguez C. Feminización en medicina: liderazgo y academia. Educ Med 2012; 15 (4): 191-195.

19. Heath I. Women in medicine: continuing unequal status of women may reduce the influence of the profession. BMJ 2004; 329: 412-3.

20. Wynn R. Saints and sinners: women and the practice of medicine throughout the ages. JAMA 2000; 283: 668-9

21. Abramo, L. y M. E. Valenzuela (2006), "Inserción laboral y brechas de equidad de género en América Latina", en Trabajo decente y equidad de género en América Latina, Chile, OIT. 